Annals of Pure and Applied Mathematics

Vol. 13, No. 1, 2017, 27-31

ISSN: 2279-087X (P), 2279-0888(online)

Published on 10 January 2017

www.researchmathsci.org

DOI: http://dx.doi.org/10.22457/apam.v13n1a3

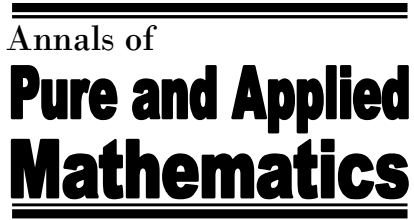

\title{
Strong Non-Split Domination in Directed Graphs
}

\author{
B.Vijayalakshmi ${ }^{1}$ and R.Poovazhaki ${ }^{2}$
}

${ }^{1}$ Department of Mathematics, Mount Carmel College, Bengaluru-560052

${ }^{2}$ E.M.G.Yadava Women's College, Madurai-625014

Email: vijiarif@yahoo.co.in; rpoovazhaki@yahoo.co.in

Research and Development centre

Bharathiar University, Coimbatore-641046

Received 20 December 2016; accepted 2 January 2017

\begin{abstract}
The concept of connectedness plays an important role in many networks. Digraphs are considered as an excellent modeling tool and are used to model many types of relations amongst any physical situations. In this paper the concept of strong non-split domination in directed graph $\mathrm{D}$ has been introduced by considering the dominating set $\mathrm{S}$ is a strong non-split dominating set if the complement of $\mathrm{S}$ is complete A dominating set $\mathrm{S}$ of a directed graph $\mathrm{D}$ is a strong non-split dominating set if the induced subdigraph $<\mathrm{V}$ $\mathrm{S}>$ is complete. The minimum cardinality of strong non-split dominating set is denoted by $\gamma_{s n s}(D)$. In this paper, the domination parameters corresponding to strong non-split domination in digraphs has been analyzed in various types of digraphs and obtained several results on these parameters.
\end{abstract}

Keywords: Tournaments, transitive tournament, Strong non-split dominating sets in digraphs

\section{AMS mathematics subject classification (2010): 05C20}

\section{Introduction}

Kulli and Janakiram introduced the concept of strong non-split domination number of a graph [10]. Throughout this paper $\mathrm{D}=(\mathrm{V}, \mathrm{A})$ is a finite directed graph with neither loops nor multiple arcs(but pairs of arcs are allowed) and $\mathrm{G}=(\mathrm{V}, \mathrm{E})$ is a undirected graph with neither loops nor multiple edges. For basic terminology on graphs and digraphs, we refer to Chartrand and Lesniak [2]

Let $G=(V, E)$ be a graph. A subset $D$ of $V$ is called dominating set of $G$ if every vertex in V-D is adjacent to at least one vertex in D. The minimum cardinality of dominating set of $\mathrm{G}$ is called domination number of $\mathrm{G}$ and is denoted by $\gamma(G)$ [6].

A dominating set $\mathrm{D} \subseteq \mathrm{V}$ of a graph $\mathrm{G}$ is non- split dominating set if the induced subgraph $\langle\mathrm{V}$-D $\rangle$ is complete. The strong non-split domination number $\gamma_{s n s}(G)$ is the minimum cardinality of a strong non-split dominating set [10]. 


\section{B.Vijayalakshmi and R.Poovazhaki}

Let $\mathrm{D}=(\mathrm{V}, \mathrm{A})$ be a digraph. A subset $\mathrm{S}$ of $\mathrm{V}$ is called a dominating set of $\mathrm{D}$ if for every vertex $v \in V-S$ there exists a vertex $u \in S$ such that $(u, v) \in A$. The domination number $\gamma(D)$ is the minimum cardinality of dominating set D [5].

Let $\mathrm{D}=(\mathrm{V}, \mathrm{A})$ be a digraph. For any vertex $\mathrm{u} \in \mathrm{V}$, the sets $\mathrm{O}(\mathrm{u})=\{\mathrm{v} /(\mathrm{u}, \mathrm{v}) \in \mathrm{A}\}$ and $\mathrm{I}(\mathrm{u})=\{\mathrm{v} /(\mathrm{v}, \mathrm{u}) \in \mathrm{A}\}$ are called outset and inset of $\mathrm{u}$. The in degree and out degree of $\mathrm{u}$ are defined by $\operatorname{id}(\mathrm{u})=|I(u)|$ and $\operatorname{od}(\mathrm{u})=|O(u)|$. The minimum in degree, the minimum out degree, the maximum in degree and maximum out degree of $\mathrm{D}$ are denoted by $\delta^{-}, \delta^{+}, \Delta^{-}$, and $\Delta^{+}$respectively. [1]

An out- domination set of digraph $\mathrm{D}$ is a set $S^{+}$of vertices of $\mathrm{D}$ such that every vertex of $\mathrm{V}-\mathrm{S}^{+}$is adjacent from some vertex of $\mathrm{S}$. The minimum cardinality of out-domination set for $\mathrm{D}$ is the out-domination number $\gamma^{+}(D)[3,5]$.

The in- domination number $\gamma^{-}(D)$ is defined as expected.

Although domination and other related concepts have been extensively studied for undirected graphs, the respective analogue on digraphs have not received much attention.

$\gamma$ - set is the set of all vertices in dominating set with \# $\gamma(D)$

$\gamma^{+}$- set is the set of all vertices in out- dominating set with \# $\gamma^{+}(D)$

$\gamma^{-}$- set is the set of all vertices in in-dominating set with \# $\gamma^{-}(D)$

$\gamma_{\text {sns }}$ - set is set of all vertices in strong non-split dominating set with \# $\gamma_{n s}(D)$

$\gamma_{\text {sns }}{ }^{+}$- set is set of all vertices in strong non-split out dominating set with \# $\gamma_{n s}{ }^{+}(D)$

$\gamma_{\text {sns }}{ }^{-}$- set is set of all vertices in strong non-split in dominating set with \# $\gamma_{n s}{ }^{-}(D)$

Throughout this paper the out-domination number of digraphs have been analyzed.

\section{Preliminaries}

Definition 1. A dominating set $\mathrm{S}$ of digraph $\mathrm{D}$ is a strong non-split dominating set if the induced sub-digraph $\langle V-S\rangle$ is complete. The minimum cardinality of strong nonsplit domination number is denoted by $\gamma_{s n s}(D)$.

Definition 2. A digraph $\mathrm{D}$ is strongly connected or strong if for every pair $\mathrm{x}, \mathrm{y}$ of distinctvertices in $\mathrm{D}$, there exists an $(\mathrm{x}, \mathrm{y})$ path and $(\mathrm{y}, \mathrm{x})$ path [7].

Definition 3. An oriented graph is a digraph with no cycle of length two [7].

Definition 4. A tournament is transitive if $(u, w)$ is an $\operatorname{arc}$ whenever $(u, v)$ and $(v, w)$ both are arcs [7].

\section{Results and observations}

For directed path $P_{n}, n \geq 2$

$\gamma_{\text {sns }}{ }^{+}(D)=n-1$. 


\section{Strong Non-Split Domination in Directed Graphs}

For directed cycle $C_{n}, n \geq 3$

$$
\gamma_{\text {sns }}{ }^{+}(D)=n-1 \text {. }
$$

For complete digraph $\gamma_{\text {sns }}{ }^{+}(D)=n-\Delta^{+}$

For directed tree, $\gamma_{\text {sns }}{ }^{+}(D)=n-1$

For directed star, $\gamma_{\text {sns }}{ }^{+}(D)=0$

$$
\gamma_{\text {sns }}{ }^{-}(D)=1 \text {. }
$$

Theorem 3.1. For any digraph $\mathrm{D}$

$$
\gamma^{+}(D) \leq \gamma_{n s}^{+}(D) \leq \gamma_{\text {sns }}{ }^{+}(D) .
$$

Proof: Let $S^{+}$be the strong non-split dominating set of digraph D. Every strong nonsplit dominating set of $\mathrm{D}$ is a non-split dominating set and every non-split dominating set is a dominating set.

Theorem 3.2. If $\mathrm{T}$ is a directed tree which is not a directed star, then $\gamma_{\text {sns }}{ }^{+}(T)=\gamma_{n s}{ }^{+}(T)=n-1$.

Proof: Since $\mathrm{T}$ is not a directed star, where directed star is a connected directed tree. Let $S^{+}$be the strong non-split dominating set which has a root vertex and cut vertices with one end vertex. The other end vertex which is in $\left\langle V-S^{+}\right\rangle$is complete.

Theorem 3.3. For any digraph $\mathrm{D}$ which is directed cycle then $\gamma_{\text {sns }}{ }^{+}(D)=n-\Delta^{+}$

Proof: Suppose $\gamma_{\text {sns }}{ }^{+}(D)>n-\Delta^{+}$

Then it must be $\gamma_{\text {sns }}{ }^{+}(D) \geq n-\Delta^{+}+1$

That is $n-\Delta^{+}+1 \leq \gamma_{\text {sns }}{ }^{+}(D)<n-\Delta^{+}$

which is a contradiction

$$
\text { Hence } \gamma_{\text {sns }}{ }^{+}(D)=n-\Delta^{+} \text {. }
$$

Theorem 3.4. Let $\mathrm{D}$ be the digraph having cut vertices then $\gamma_{\text {sns }}{ }^{+}-$set of $\mathrm{D}$ would contain all cut vertices.

Proof: Let $v$ be a cut vertex and $S^{+}$is a $\gamma_{s n s}{ }^{+}$- set of D. If $v \notin S^{+}$then $D-v$ has exactly two components $D_{1}$ and $D_{2}$ such that at least one of the digraphs $H_{1}=<D_{1} \bigcup\{v\}>$ or $H_{2}=<D_{2} \bigcup\{v\}>$ is a path or directed cycle.

Theorem 3.5. Let $K_{1, p}$ be a directed star in which $\operatorname{od}\left(\mathrm{v}_{\mathrm{i}}\right)=\mathrm{p}$ and $\operatorname{id}(\mathrm{v})=1,1 \leq i \leq p$ Then $\gamma_{\text {sns }}{ }^{+}(D)=0$. 


\section{B.Vijayalakshmi and R.Poovazhaki}

Proof: As $\left\langle V-S^{+}>\right.$contains the vertices $v_{1}, v_{2}, v_{3}$ and $S^{+}=\{v\}$ then $<V-S^{+}>$is not connected and complete. Hence there is no strong non-split domination number as well as non-split domination number.

$$
\therefore \gamma_{\text {sns }}{ }^{+}(D)=\gamma_{n s}{ }^{+}(D)=0
$$

Theorem 3.6. Let $K_{1, p}$ be a directed star in which $\operatorname{od}\left(\mathrm{v}_{\mathrm{i}}\right)=1,1 \leq i \leq p$ and $\mathrm{id}(\mathrm{v})=\mathrm{p}$ Then $\gamma_{\text {sns }}{ }^{+}(D)=1$

Proof: Let $S^{+}=p=\left\{v_{1}, v_{2}, v_{3}\right\}$ and $\left\langle V-S^{+}\right\rangle=1$ which is connected and complete, then $\gamma_{\text {sns }}{ }^{+}(D)=\{v\}=1$.

Theorem 3.7. Let $l(D)$ denotes the length of largest directed path then $\gamma_{\text {sns }}{ }^{+}(D)=l(D)$.

Proof: Let $\mathrm{P}=\left\{v_{0}, v_{1}, \ldots . v_{k}\right\}$ be a largest path in $\mathrm{D}$ with $\mathrm{k}=l(D)$. Let $\mathrm{S}$ be the strong non-split dominating set of $\mathrm{D}$.

As $V-S$ has only one vertex with od=0 and id=1.

Therefore $V-S$ is complete. Hence $\mathrm{S}$ has all the vertices except that vertex which has od $=0$ and id $=1$ then $\gamma_{\text {sns }}{ }^{+}(D)=l(D)$.

Theorem 3.8. If $\mathrm{D}$ is the tournament digraph then ${\gamma_{s n s}}^{+}(D)=n-\Delta^{+}$.

Proof: Suppose $D$ is a tournament digraph which has vertices $n=4$

Case (i) : Let $V-S=\{\mathrm{u}, \mathrm{v}, \mathrm{w}\}$ be complete, $\mathrm{S}$ has only one vertex 'x' which dominates $\mathrm{u}, \mathrm{v}$, and $\mathrm{w}$, then the maximum out degree of $\mathrm{D}$ is 3. Hence $\gamma_{\text {snn }}{ }^{+}(D)=n-\Delta^{+}$.

Case (ii) : Let $V-S=\{\mathrm{u}, \mathrm{v}\}$ be complete, $\mathrm{S}=\{\mathrm{x}, \mathrm{w}\} \mathrm{x}$ dominates $\mathrm{u}$ and $\mathrm{v}$ or $\mathrm{w}$ dominates $\mathrm{u}$ and $\mathrm{v}$. then the maximum out degree of $\mathrm{D}$ is 2 . Hence $\gamma_{\text {sns }}{ }^{+}(D)=n-\Delta^{+}$.

After analyzing both the cases, for $\mathrm{n}=4$.we can conclude in general for any tournament digraph $\gamma_{s n s}^{+}(D)=n-\Delta^{+}$.

\section{Conclusion}

In this paper, we have introduced the parameter strong non-split domination in directed graphs. Some interested results related with the above are proved. Further, the authors proposed to introduce new dominating parameters in directed graphs using the MATLAB codes. In the last decade, we have seen an impressively increasing number of neuroscience studies using digraph theory and networks. Neural networks are considered as directed graphs that allows a broad range application of analytical tools from digraph theory. 


\section{Strong Non-Split Domination in Directed Graphs}

\section{REFERENCES}

1. S.Arumugam, K.Jacob and Lutz Volkmann, Total and connected domination in digraphs, Australian Journal of Combinatorics, 39 (2007) 283-292

2. G.Chartrand and L.Lesniak, Graphs and Digraphs, CRC Press, 2004.

3. G.Chartrand, F.Harary and B.G.Yu,On the out-domination and in-domination of a digraph, Discrete Math. 197/198 (1999) 179-183.

4. E.J.Cockayne, Domination of undirected graphs. A survey in theory and Applications of Graphs, Lecture Notes in Mathematics, 642 (1978)141-147.

5. J.Ghosal, R.Laskar and D.Pillone, Topics on domination in direceted graphs.

6. T.W.Haynes, S.T.Hedetniemi and P.J.Slater, Domination in Graphs-Advances Topics, Marcel Dekker Inc., New York, 1998.

7. W.Haynes, S.T.Hedetniemi and P.J.Slater, Fundamentals of domination in graphs, Marcel Dekker Inc., New York, 1998.

8. Jorgen Bang-Jensen, Gregory Gutin, Digraphs Theory, Algorithms and Applications, 15th August 2007

9. V.R.Kulli and B.Janakiram, The split domination number of a graph, Graph Theory Notes of New York, New York Academy of Sciences XXXII(1997)16-19.

10. V.R.Kulli and B.Janakiram, The non-split domination number of a graph, Indian $J$ Pure Appl. Math., 31(5) (2000) 545-550.

11. V.R.Kulli and B.Janakiram, The strong non-split domination number of a graph. International Journal Management and Systems, 19(2) (2003) 145-156.

12. B.Vijayalakshmi and R.Poovazhaki, Point-set domination in directed graphs, International Journal of Mathematical Archieve, 6(10) (2015) 82-87.

13. B.Vijayalakshmi and R.Poovazhaki, Split domination in directed graphs, International Journal of Mathematical Archieve, 6(11) (2015) 142-145.

14. B.D.Acharya and K.A.Germina, Set-valuations of graphs and their applications: a survey, Annals of Pure and Applied Mathematics, 4(1) (2013) 8-42 .

15. M.Pal, Intersection graphs: an introduction, Annals of Pure and Applied Mathematics, 4(1) (2013) 43-91.

16. N.Saradha and V.Swaminathan, Strong complementary acyclic domination of a graph, Annals of Pure and Applied Mathematics, 8(2) (2014) 83-89. 